\title{
Interference and dominance in texture segregation: Hue, geometric form, and line orientation
}

\author{
TARA C. CALLAGHAN \\ St. Francis Xavier University, Antigonish, Nova Scotia, Canada
}

\begin{abstract}
Five experiments were designed to test whether (1) lowering the similarity of elements within a region of texture (low-similarity arrays) would interfere with texture segregation, and (2) there would be dominance of one type of property difference over another in determining an observer's choice of boundary in two-boundary (ambiguous) displays. In Experiments 1 and 2, the interference question was assessed using stimuli formed from the dimensions hue and geometric form (circle/square or straight/curved novel shapes). The results indicated that when boundary judgments were based on form differences, segregation was significantly impaired by hue variation. However, hue segregations were not affected by form variation. In Experiments 3-5, the dominance question was examined using stimuli formed from hue and geometric form, as well as those formed from hue and line orientation (horizontal/vertical)! Analyses revealed that there was no dominance of one type of property difference over another. Rather, observers' performance was completely predicted by the relative discriminabilities of the two boundaries. These findings support Beck's (1982) model of textural segmentation and call into question traditional notions of the preattentive stage of perceptual processing.
\end{abstract}

The perception of separate regions of texture in the visual field is a process that proceeds automatically, with little or no apparent effort. In the phenomenological experience of casually scanning the real visual world, an observer is aware of vague regions of difference and hazy boundaries between indistinct forms. The precise properties of these forms-their hue, shape, orientation, spatial location, or pattern of movement-are not directly accessible to the observer in this casual state. However, given the requisite motivation (e.g., "Find your red-headed son in that mass of children"), the observer is quickly shifted into a directed state of perception, now able to "zoom in" on a specific property, or combination of properties, to the exclusion of others.

This description of the phenomenon of perception fits nicely with the early feature-integration theory of object perception proposed by Treisman (Treisman \& Gelade, 1980; Treisman \& Paterson, 1984; Treisman \& Souther, 1985). In that view, the automatic grouping together of

\footnotetext{
Experiments 1 and 3 were conducted at Yale University, where the author was supported by a Natural Sciences and Engineering Research Council of Canada postdoctoral fellowship, and additional support from National Institute of Mental Health Grant MH 14229 to W. R. Garner. The remaining experiments were conducted at St. Francis Xavier University and were supported by a Natural Sciences and Engineering Research Council of Canada operating grant to the author. The author thanks W. R. Garner, Richard Ivry, and two anonymous reviewers for their helpful comments on an earlier draft of this paper. Thanks also to Annette MacArthur for help in running some of these experiments. Requests for reprints should be sent to Tara C. Callaghan, Department of Psychology, St. Francis Xavier University, Antigonish, Nova Scotia B2G 1 C0 Canada.
}

similar elements, and separation of them from dissimilar ones, is allocated to an early, preattentive stage of perceptual processing. During preattentive processing, parallel and independent registration of features from across the visual field occurs. These features are registered on separate "maps" that are linked to a master location map. When detailed processing of an object of perception is required, for example when one identifies an object, a further stage of attentional analysis is accessed. It is proposed that focused attention allows for the linking together of information found in given spatial locations across the master map. The early version of the theory implied that the shift from independence to interdependence of features is tantamount to a shift from preattentive to attentive processing.

In a recent modification of the feature-integration theory, Treisman and Gormican (1988) have abandoned the dichotomy view of preattentive/attentive stages and now see attention as varying along a continuum during perceptual processing. Thus, attention can range from widely dispersed, as in texture segregation, to sharply focused on a single item, as in visual search for a conjunction of features in a heterogeneous background of elements that share those features. It is clear that the main objectives of the revised feature-integration theory are to establish the primitive features of visual perception (see next section for details), to describe how these features are initially coded by the visual system (independent feature maps linked to a master location map), and to determine how the initial independent registration of features is related to the conscious experience of object perception (narrowing of the focus of attention to a discrete spatial 
location). With the elimination of the dichotomy view of attention, many of the previously discrepant findings from texture-segregation research (e.g., Beck, 1982; Callaghan, 1984; Callaghan, Lasaga, \& Garner, 1986) may now fit nicely in the revised feature-integration view of perception.

\section{Visual Primitives in Texture Segregation}

The view that visual texture segregation is a process that is accomplished relatively early in perception, and with a widely dispersed focus of attention, is shared by many researchers (Beck, 1972, 1982; Khaneman, 1973; Neisser, 1967; Treisman \& Gelade, 1980; Treisman \& Gormican, 1988). Studies of texture segregation have focused on the determination of the basic properties of the stimulus that mediate segregation (i.e., visual primitives), as well as on the illumination of the nature of the segregative process itself. From the former studies, and from studies using a visual-search pop-out paradigm, we have learned that texture segregation and popout are easily produced by the properties of line orientation (Beck, 1966, 1967, 1972; Callaghan et al., 1986; Olson \& Attneave, 1970; Treisman \& Gormican, 1988), hue (Callaghan, 1984; Treisman, 1982; Treisman \& Gelade, 1980; Treisman \& Gormican, 1988), brightness (Callaghan, 1984; Treisman \& Gormican, 1988), or form (Treisman, 1982; Treisman \& Gelade, 1980; Treisman \& Gormican, 1988). There is indication that the presence versus absence of line terminators (Julesz, 1975, 1981; Treisman \& Souther, 1985), line length (Beck, 1974; Treisman \& Gormican, 1988), and curvature (Treisman \& Gormican, 1988), as well as the property of closure (Treisman \& Paterson, 1984) also promote strong segregation. With the exception of recent evidence (Enns, 1986; Treisman \& Souther, 1985) suggesting that the effectiveness of line terminators and closure in mediating segregation can be limited in certain contexts, there has been general agreement on the status of these properties as primitives in visual perception, based on the convergence of findings from segregation and visual-search studies.

\section{Within-Region Similarity and \\ Between-Region Differences}

A second focus of texture-segregation research has been to examine the processes by which features group together to form larger regions of texture. On the basis of the Gestalt notion of similarity grouping, it has been proposed that the process of texture segregation will be enhanced not only by high dissimilarity of elements between regions, but also by high similarity of elements within regions (Beck, 1982; Callaghan et al., 1986; Enns, 1986; Olson \& Attneave, 1970). Although much research has been devoted to the study of between-region differences, at least two lines of evidence support the importance of withinregion differences.

In one line of investigation (Callaghan, 1984; Callaghan et al., 1986), it has been shown that if the similarity of elements within a region is reduced by randomly varying the level of an irrelevant dimension across all elements in the array, then performance is poor relative to a control array having identical elements within a region. In a second line of research, Enns (1986) has reported that ease of segregation is determined by the context in which a target region is embedded. In Enns's study, texture segregation was completely eliminated when the salience of the unique element differences between regions was low, relative to the similarities of those elements across the whole array.

If one considers search for a single target in the visualsearch task to be a special case of texture segregation (or vice versa), there may be a third source of support (albeit tangential) for the role of within-region differences from recently reported visual-search asymmetries (Treisman \& Gormican, 1988; Tresiman \& Souther, 1985). For example (Treisman \& Souther, 1985, Experiment 1), a circle with an intersecting line will easily segregate from a background of plain circles, but a plain circle will not pop out from a background of circles with intersecting lines. Treisman and Gormican (1988) have provided a formal explanation, based on Weber's law, to account for the intradimensional asymmetries. Although it would not challenge the qualification of these features for the status of visual primitives, it would be interesting for the present purposes to see whether the asymmetry noted by these researchers would be eliminated in a task that required observers to locate a discrepant quadrant of elements in an array. If segregation was obtained in the quadrant task, then the facilitatory effects of within-region similarity would be supported. Assessment of the significance of the visual-search asymmetries to the question of withinregion similarity in texture segregation will depend on further research.

\section{Purpose of the Present Experiments}

The primary aim of the present experiments was to provide further support for the proposal that within-region similarity is an important factor in texture segregation (Beck, 1982; Callaghan et al., 1986; Enns, 1986; Olson \& Attneave, 1970). The procedure established by the author (Callaghan, 1984) was employed in the present experiments with stimuli that varied on the dimensions of hue and geometric form. In the original procedure, observers were presented with 36-element arrays that contained either a vertical or a horizontal boundary. A single property difference defined the regions of texture. A second property was either held at a constant level for all elements in the array (control arrays) or was alternated irrelevantly across the array (orthogonal arrays). Callaghan et al. (1986) noted that in orthogonal arrays, the similarity of elements within each of the two regions of texture is reduced relative to control arrays. This manipulation produced strong interference of segregation that was symmetric for hue and brightness (Callaghan, 1984) and for hue and horizontal/vertical or left/right diagonal orientations (Callaghan et al., 1986), and was asymmetric for hue and horizontal/left diagonal orientation (Callaghan et al., 1986) stimuli. It was expected that this procedure 
would also produce interference of segregation when hue and geometric form were varied in the stimulus arrays of Experiments 1 and 2.

Two lines of evidence support this prediction. Pashler (1988) asked observers to locate (left or right side of a 90-element matrix) a single target embedded in a background of elements. Although within-region differences could not operate in this procedure (search was for a single target element), there was some indication that when observers were searching for a target that differed in form (0 vs. /) from background elements and the hue of those background elements varied irrelevantly (red vs. green), search was impaired (Experiment 4). Search was not affected when observers searched for a hue difference among distractors that varied in form (Experiment 3). The asymmetric interference was not found in all experiments. Pashler's use of forms ( 0 vs. /) that varied in other attributes found to be meaningful in early perception (i.e., area, curvature, line terminators, closure) makes it difficult to assess why interference occurred in his experiments. Furthermore, cross-experiment comparison suggests that the hues used in his experiments were easier to discriminate than were the forms; thus, any asymmetric interference may simply have been due to discriminability differences (Garner, 1983).

A second line of research that predicts hue/form asymmetry comes from the attentional literature (Garner, 1983; Gottwald \& Garner, 1972; Schroeder, 1976). Garner and his colleagues have repeatedly shown that selective attention to hue when form (circle, square) varies irrelevantly is easier than selective attention to form when hue varies. If asymmetry was also found in this texture-segregation task, it was expected to be in the same direction as that found in studies of attention.

A second aim of the present experiments was to establish a procedure that could tackle an issue that has not yet been broached by investigators of early perception. That is, what happens when one makes the array more complex, more like the real-world experience of finding a boundary in a multidimensional array? In the real world, we are not usually given a single property difference on which to base our decision; rather, we are faced with differences in many properties, and often the boundaries that would be predicted from these separate sets of differences are themselves different. The question that was posed in Experiments 3-5 was whether some property differences dominate others in an observer's decision of where the boundary lies in a textured array.

In Experiments 3-5, observers were presented with arrays that contained two potential boundaries, one defined by the property of hue, and the other by the property of form (Experiments 3 and 4 ) or line orientation (Experiment 5). The observers' task was simply to indicate whether the boundary was horizontal or vertical (Experiments 3 and 4) or to locate the discrepant quadrant (Experiment 5), as in the earlier experiments. On the basis of the research that has provided information regarding visual primitives, one would expect that there should be no dominance of one property over the other early in processing; all visual primitives should be equally primitive. However, given Treisman's recent findings (Treisman \& Gormican, 1988; Treisman \& Souther, 1985) of withindimension search asymmetries, we may expect to find that, in certain combinations, one property does in fact dominate in this task, although more specific predictions for the present stimuli, which varied between dimensions, cannot be made from their results.

\section{EXPERIMENT 1}

In this experiment, the importance of within-group similarity to visual texture segregation was assessed using a task devised by the author (Callaghan, 1984) and stimuli formed from hue and geometric form (circle, square) differences. It was expected that reducing the similarity of elements within regions of texture would impair performance and, furthermore, that if there was asymmetry, irrelevant hue variation would produce stronger interference than would form variation.

\section{Method}

\section{Observers}

Sixteen Yale undergraduates were either paid or received course credit for participation in this experiment. All participants had normal or corrected vision. Each observer took part in one experimental session that lasted approximately $1 \mathrm{~h}$.

\section{Procedure}

On each trial, observers were presented with a 36-element stimulus array. Each stimulus array contained two regions that were implicitly separated by a horizontal $(H)$ or a vertical (V) boundary. Sample arrays are shown in Figure 1. Reaction time (RT), in milliseconds, from the onset of the stimulus until the observer pressed a button to indicate the nature of the boundary (i.e., $\mathrm{H}$ or $\mathrm{V}$ ), was the dependent measure.

The experimenter initiated a trial after a verbal "ready" signal. Upon initiation of a trial, the stimulus array was presented for $1 \mathrm{sec}$. The observer responded as quickly as possible by pressing, with the index finger of his or her preferred hand, one of two response buttons that had been arbitrarily designated as $\mathrm{H}$ or $\mathrm{V}$ by the experimenter. Between trials, the observer's index finger rested on a button that was equidistant from the two response buttons.

\section{Stimulus Construction and Presentation}

The stimuli were $35-\mathrm{mm}$ slides of $6 \times 6$ matrices (i.e., 36-element arrays) composed of elements that varied in hue and geometric form. Three hues, all from the Munsell color system, were used: $\mathrm{Hl}$ (10R 4/12), H2 (7.5RP 4/12), and H3 (2.5R 4/12). Two forms were used: circle and square. Templates of form arrays were first cut into thin, black cardboard. Then the Munsell color chips were positioned behind the appropriate template and slides were taken.

Stimuli were presented in a Gerbrands random-access projection tachistoscope (Model G1177). The slides were rear-projected onto a screen that had response buttons mounted on a lower panel. Depression of the response buttons activated a digital timer (Gerbrands G1271) and the experimenter recorded RT and errors. Approximate viewing distance was $50 \mathrm{~cm}$. The viewing size of in- 


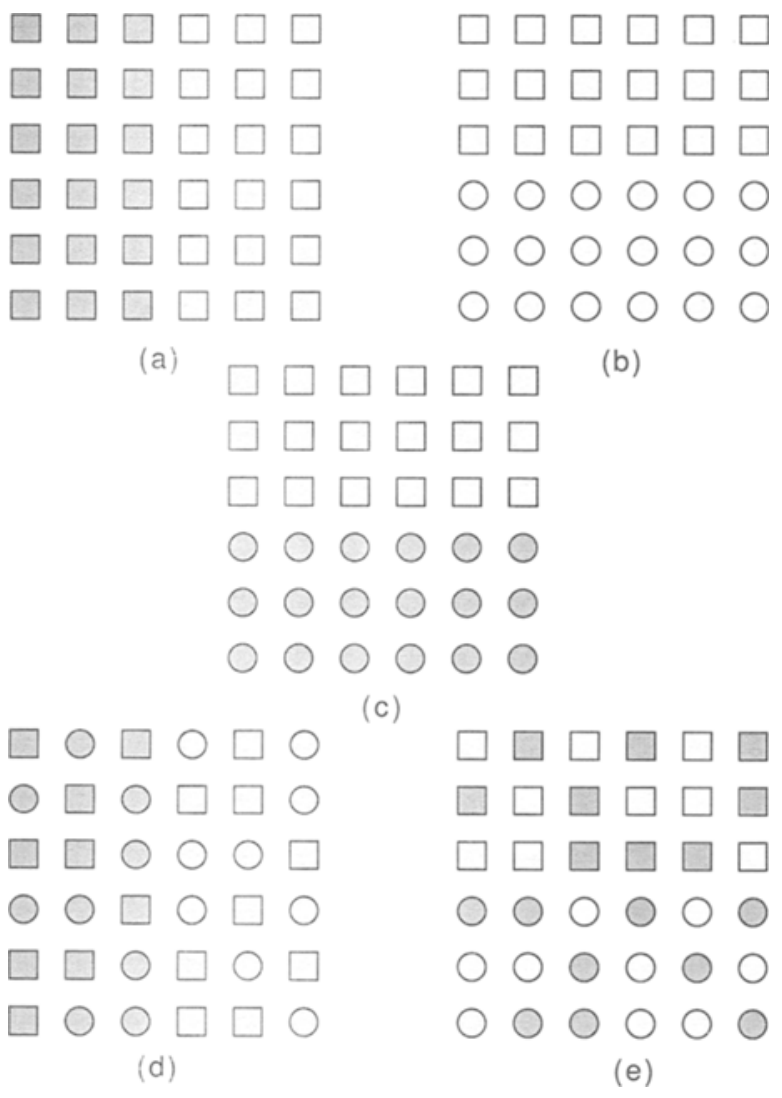

Figure 1. Examples of textured arrays containing boundaries defined by hue differences (represented by filled and unfilled forms) for (a) control and (d) low-similarity arrays, by form differences (circle vs. square) for (b) control and (e) low-similarity arrays, and by both differences for (c) redundant arrays.

dividual elements was approximately $0.5^{\circ}$ of visual angle, and of the entire array approximately $5.7^{\circ}$ on each side. Observers were tested in a dark room.

\section{Stimulus Conditions}

The stimulus arrays for this experiment were of two major types.

Single-property arrays (control and low-similarity). Each single-property array allowed for segregation according to either hue or form. Hue arrays differed in the particular hue difference that produced texture. For "easy" arrays, the hue difference was 5 steps in the Munsell system (H1 vs. H2), and for "hard" arrays, the difference was 3 steps (H1 vs. H3). All form arrays contained a boundary defined by a difference of circle versus square elements.

For each array, segregation could be based on one property difference only, either hue or form; however, the other property in the array could be either held constant (control arrays) or varied randomly (low similarity). ${ }^{1}$

In hue control arrays (Figure la), levels of hue were varied and the level of form was held constant at one of two levels (circle or square). There were two possible subtypes of hue control arrays that corresponded to the discriminability of the hue difference defining the boundary: easy (5-step) and hard (3-step). For each of these possibilities, half of the arrays contained circle elements and half contained square elements. Preliminary analyses indicated that the particular shape of elements did not influence RT for hue control arrays in this and subsequent experiments; thus, in all further analyses, data were combined across the particular form level used.
For form control arrays (Figure 1b), the property of form was varied and that of hue was held constant across all elements in the array. There was only one type of form control array (i.e., containing a circle vs. square difference across the boundary). However, to control for any possible effects due to the particular hue of the elements in these arrays, one third of the form control arrays contained elements with the $\mathrm{H} 1$ level, one third $\mathrm{H} 2$, and one third $\mathrm{H} 3$. Preliminary analyses revealed that in this and in subsequent experiments, the specific hue used (i.e., H1, H2, or H3) had no effect on RT for form control arrays. Thus, these data were combined for all further analyses.

For hue low-similarity arrays (Figure 1d), differences in hue produced texture, but the two levels of form varied in a random manner. For half of these arrays, the hue differences were easy to discriminate (hue easy-low similarity), and for half they were hard to discriminate (hue hard-low similarity).

In form low-similarity arrays (Figure le), differences in form produced texture, but levels of hue varied across the array. For half of these arrays, the hue differences that were varied irrelevantly were easy to discriminate (form easy-low similarity), and for half they were hard to discriminate (form hard-low similarity).

Two-property (redundant) arrays. The second type of stimulus array (Figure 1c) was one in which texture segregation could be based on either one or both property differences (i.e., hue and/or form). This redundant combination of hue (easy or hard) and form differences resulted in two types of arrays: hue easy-redundant and hue hard-redundant.

\section{Experimental Design}

Forty different stimulus arrays (20 using easy hue levels, and 20 using hard hue levels) were used in this experiment. Sixteen of these arrays could be segregated on the basis of hue differences alone ( 8 control and 8 low-similarity), 16 on the basis of form alone ( 8 control and 8 low-similarity), and 8 on the basis of a redundant combination of hue and form. When hue differences produced texture, half of the arrays contained an easy hue difference and half contained a hard hue difference. When hue differences were varied irrelevantly (i.e., form low-similarity), half of the arrays contained an easy hue variation (form easy-low similarity) and half contained a hard hue variation (form hard-low similarity).

Each observer was presented with 11 blocks of trials, with the first block considered as practice. In each block, all $\mathbf{4 0}$ possible stimuli were presented once and the order of presentation was randomized.

\section{Results and Discussion}

The error rate was low (overall mean proportion of errors $=.03$ ) and there was no evidence of a speed/ accuracy tradeoff. Therefore, only RTs for correct responses were submitted to analysis. The raw RT data for each observer's correct responses were combined across blocks for each array subtype, and median RTs were then calculated for these arrays.

\section{Control Analyses}

To establish the relative discriminability of the two component dimensions used in these arrays (i.e., hue and form), a one-way analysis of variance (ANOVA) of all control data (i.e., hue easy-control, hue hard-control, and form control) was conducted. There was a significant main effect of control array $[F(2,30)=5.98, p<.006]$. Post hoc analyses revealed that RTs were equivalent for form control (571 msec) and hue easy-control $(567 \mathrm{msec})$ arrays, and that both of these were faster $(p<.01)$ than 
those for hue hard-control arrays $(592 \mathrm{msec})$. Interference and redundancy effects will be interpreted in light of these relative discriminabilities.

\section{Interference Analyses}

Hue arrays. To analyze the interference produced by random variation of form levels on hue segregation, a subjects $\times$ hue discriminability (easy, hard) $\times$ subtype of stimulus array (control, low-similarity) ANOVA was conducted (see Table 1 for means corresponding to this analysis). The only significant effect was a main effect of hue discriminability $[F(1,15)=16.73, p<.001]$. In line with the results reported above, post hoc analyses revealed that mean RT for easy hue arrays was faster $(p<.01)$ than that for hard hue arrays. There was no effect of subtype of stimulus array. Thus, random variation of form levels did not interfere with hue segregation, even when the form differences present in the array were easier to discriminate than the hue differences (i.e., hue hard-low similarity). This finding is consistent with the findings of Pashler (1988, Experiment 3), and with reports from the attentional literature (Garner, 1983; Gottwald \& Garner, 1972; Schroeder, 1976) suggesting that hue information may be processed with little or no interference from irrelevant form variation. It also supports Treisman and Gelade's (1980) claim that hue and form properties are independently registered in preattention. Finally, the notion that within-region similarity has an important impact on segregation is qualified by these results. When withinregion similarity is reduced by the random variation of form elements, hue segregation proceeds unimpeded, both when hue and form differences are equivalent (hue easylow similarity) and when hue is harder to discriminate than form (hue hard-low similarity).

Form arrays. To assess the interference of hue variation on the observers' ability to segregate form arrays, a subjects $\times$ hue discriminability (easy, hard) $\times$ subtype of stimulus array (control, low-similarity) ANOVA was conducted (see Table 2 for mean RTs relative to this analysis). This analysis revealed a significant main effect of

Table 1

Mean Reaction Times (in msec) for Correct Segregation Responses in the Interference Analysis of Hue Arrays in Experiment 1

\begin{tabular}{cccc}
\hline Hue & \multicolumn{3}{c}{ Stimulus Array } \\
\cline { 2 - 4 } Discriminability & Control & Low-Similarity & Average \\
\hline Easy & 567 & 571 & 569 \\
Hard & 592 & 592 & 592 \\
Average & 580 & 582 & 581 \\
\hline
\end{tabular}

Table 2

Mean Reaction Times (in msec)

for Correct Responses in the Interference Analysis

of Form (circle/square) Arrays in Experiment 1

\begin{tabular}{cccc}
\hline $\begin{array}{c}\text { Hue } \\
\text { Discriminability }\end{array}$ & Control & Low-Similarity & Average \\
\hline Easy & 571 & 588 & 580 \\
Hard & 571 & 587 & 579 \\
Average & 571 & 588 & 580 \\
\hline
\end{tabular}

Table 3

Mean Reaction Times (in msec) for Correct Segregation Responses in the Analysis of Redundancy Effects in Experiment 1

\begin{tabular}{ccc}
\hline $\begin{array}{c}\text { Hue } \\
\text { Discriminability }\end{array}$ & Stimulus Array \\
\cline { 3 - 3 } Easy & Redundant & Fastest Control \\
Hard & 559 & 558 \\
\hline
\end{tabular}

subtype $[F(1,15)=8.74, p<.01]$. Mean $\mathrm{RT}$ for lowsimilarity arrays was longer than that for control arrays, both when the levels of hue varied were easy to discriminate (form easy-low similarity) and when they were hard to discriminate (form hard-low similarity). Now the reduction of element similarity within a region did interfere with segregation. This finding for form arrays supports Pashler's (1988, Experiment 4) work and the attentional experiments mentioned earlier. It does not support Treisman and Gelade's (1980) claim for independent registration of features in preattention. However, the revised feature-integration theory (Treisman \& Gormican, 1988) may accommodate this finding by suggesting that the attentional focus has begun to narrow in the segregation task, thus allowing for a preliminary combination of feature information.

The asymmetry of interference found for hue and geometric form dimensions may be indicative of a privileged status for hue differences, relative to the form differences used here (i.e., circle vs. square), in producing texture segregation. This notion will be tested in Experiment 3. In addition, the data for form arrays supports the proposal that within-region similarity has an important role in the process of segregation. However, the particular manner by which the similarity of elements within a region is reduced (i.e., by variation of hue or form differences) seems to have an important influence on whether interference of segregation is exhibited.

\section{Redundancy Analyses}

To analyze redundancy-gain effects, each observer's median RT for each type of redundant array (hue easyredundant and hue hard-redundant) was compared with the faster of his/her median control RTs in $t$ tests for matched groups. This procedure was used because individual observers differed in their RTs to the two dimensions, and a redundancy gain may be inferred only if RT to the redundant dimensions is faster than RT to either of the component dimensions (see Table 3 for mean RTs corresponding to these analyses).

The results of the $t$ tests revealed that there were no gains in segregation performance when hue and form dimension differences were correlated within a single array. This finding is consistent with the pattern of results reported for hue and form dimensions in attentional studies. Furthermore, an implication of Beck's (1982) model is that texture segregation will be enhanced when betweenregion differences increase. The present results suggest that this view needs to be qualified. Hue and geometric form differences were not additive in this preattentive task. 
Nonadditivity is consistent with Treisman and Gelade's (1980) model.

\section{EXPERIMENT 2}

In this experiment, an attempt was made to replicate the findings of Experiment 1 using different geometric forms to construct the stimuli. Here, two novel forms replaced the prototypical forms of circle and square. The novel forms (see Figure 2) differed in the property of curvature. Both forms shared the property of symmetry and were of equal area. ${ }^{2}$ If the findings of Experiment 1 were replicated using the novel forms, then it would suggest that the stimulus property that was operating to produce texture segregation with the geometric form variations of Experiments 1 and 2 was curvature.

\section{Method}

All aspects of the procedure, stimulus construction and presentation, and experimental design were identical to those of Experiment 1 , with the only exception being the particular forms (straight, curved) that were used to create the arrays.

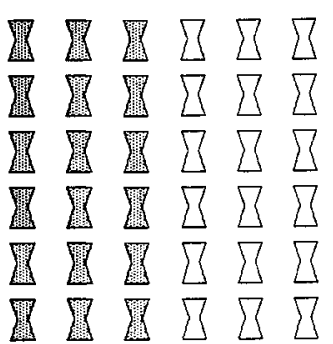

(a)

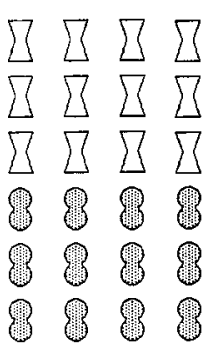

(c)

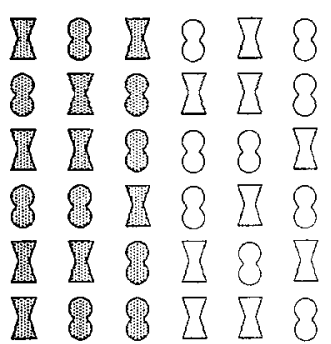

(d)

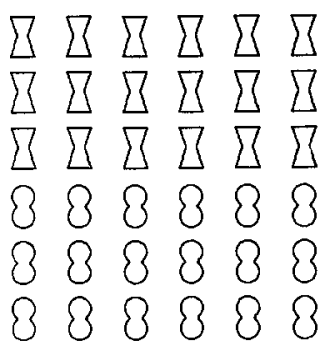

(b)

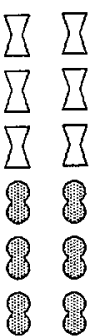

\section{8
8
8
8
8}

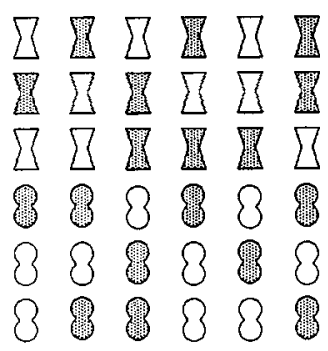

(e)

Figure 2. Examples of textured arrays containing boundaries defined by hue differences (represented by filled and unfilled forms) for (a) control and (d) low-similarity arrays, by form differences (straight vs. curved) for (b) control and (e) low-similarity arrays, and by both differences for (c) redundant arrays.
Table 4

Mean Reaction Times (in msec) for Correct Segregation Responses in the Interference Analysis of Hue Arrays in Experiment 2

\begin{tabular}{cccc}
\hline Hue & \multicolumn{3}{c}{ Stimulus Array } \\
\cline { 2 - 4 } Discriminability & Control & Low-Similarity & Average \\
\hline Easy & 707 & 728 & 718 \\
Hard & 743 & 792 & 768 \\
Average & 725 & 760 & 743 \\
\hline
\end{tabular}

\section{Observers}

Twelve St. Francis Xavier undergraduates were paid for their participation in this experiment. All participants had normal or corrected vision. Each observer took part in one experimental session that lasted approximately $1 \mathrm{~h}$.

\section{Results and Discussion}

The error rate was low (overall mean proportion of errors $=.03$ ) and there was no evidence of a speed/ accuracy tradeoff. Therefore, only RTs for correct segregation responses were analyzed. As in Experiment 1, the raw RT data for each observer's correct responses were combined across blocks for each array subtype, and median RTs were then calculated and entered into analyses.

\section{Control Analyses}

To assess the relative discriminability of the component dimensions, hue and form (straight, curved), a one-way ANOVA of all control data (i.e., hue easy-control, hue hard-control, and form control) was conducted. There was a significant main effect of control array $[F(2,22)=10.99$, $p<.005]$. Post hoc analyses revealed that RTs for form control arrays $(755 \mathrm{msec})$ were equivalent to those for hue hard-control $(743 \mathrm{msec})$, and that RTs for these were, in turn, longer $(p<.01)$ than those for hue easy-control $(707 \mathrm{msec})$. Interference and redundancy effects will be interpreted in light of these relative discriminabilities.

\section{Interference Analyses}

Hue arrays. To analyze the effects of interference due to the random variation of the novel form elements, a subjects $\times$ hue discriminability (easy, hard) $\times$ subtype of stimulus array (control, low-similarity) ANOVA was conducted (see Table 4 for relevant means). As in Experiment 1 , the only effect was a main effect of hue discriminability. This effect indicated that mean RT was significantly faster $(p<.01)$ for easy, relative to hard, hue arrays. Thus, in this experiment, as in Experiment 1, the geometric form variation was not sufficient to produce interference of segregation when boundaries were defined by hue differences. This occurred in the present experiment even when form differences were equal in discriminability, as indicated by control-array data, to hue differences (i.e., hue hard-low similarity).

Form arrays. To assess whether hue variation interfered with the segregation of form arrays, a subjects $x$ hue discriminability (easy, hard) $X$ subtype of stimulus array (control, low-similarity) ANOVA was performed 
(see Table 5 for means relevant to this analysis). This analysis revealed main effects of hue discriminability and subtype of stimulus array. These main effects are qualified by a significant two-way interaction $[F(1,11)=9.35$, $p<.01]$. Post hoc analyses of this interaction revealed that mean RT for low-similarity arrays was longer than that for control arrays at both levels of hue discriminability, but that the interference effect was stronger for form hard-low similarity $(p<.01)$ than for form easy-lowsimilarity $(p<.05)$ arrays.

Taken together with the findings of Experiment 1, these results suggest that variation of hue within a region of texture can provide strong interference when the observer must make a boundary judgment on the basis of form differences, whether those form differences are between prototypical shapes or novel ones. Beck's (1982) model is partially supported by these findings. However, there is no mechanism in his model to explain why hue differences, but not form differences, produce interference when the two component dimensions are of equal discriminability. As suggested earlier, it may be the case that certain dimensions dominate the observer's preattentive perceptual world and are given privileged status when within-region similarity is calculated. The question of dimensional dominance will be addressed in the next three experiments.

\section{Redundancy Analyses}

To assess redundancy gains when hue and form differences were correlated in arrays, each observer's median RT for redundant arrays was compared with the faster of his/her median control RT using correlated $t$ tests (see Table 6 for relevant data). These analyses revealed that there were no gains in performance when hue differences were easy to discriminate, but that mean RT for redundant arrays was significantly faster $[t(11)=16.73$, $p<.01]$ than that for the fastest control when hue differences were hard to discriminate. Thus, these results indicate that hue and the novel form differences are addi-

Table 5

Mean Reaction Times (in msec) for Correct Responses in the Interference Analysis of Form Arrays in Experiment 2

\begin{tabular}{cccc}
\hline Hue & \multicolumn{3}{c}{ Stimulus Array } \\
\cline { 2 - 4 } Discriminability & Control & Low-Similarity & Average \\
\hline Easy & 755 & 770 & 763 \\
Hard & 755 & 814 & 785 \\
Average & 755 & 792 & 774 \\
\hline
\end{tabular}

Table 6

Mean Reaction Times (in msec) for Correct Segregation Responses in the Anslysis of Redundancy Effects in Experiment 2

\begin{tabular}{ccc}
\hline Hue & \multicolumn{2}{c}{ Stimulus Array } \\
\cline { 2 - 3 } Discriminability & Redundant & Fastest Control \\
\hline Easy & 701 & 705 \\
Hard & 695 & 735 \\
\hline
\end{tabular}

tive, but only when the task is fairly difficult (i.e., when hue differences are hard to discriminate). The finding that increasing between-region dissimilarity in redundant arrays serves to ease the segregation response fits with predictions made from Beck's (1982) model.

\section{EXPERIMENT 3}

In this experiment, an attempt was made to complicate the usual experimental task of texture segregation and to get closer to real-world experience by presenting observers with arrays that contained either one or two boundaries between the regions of texture. The stimulus arrays for this experiment were formed using the same dimensional differences employed in Experiment 1. The specific question that was addressed was: Will hue differences dominate over form (circle, square) differences when the observer has to choose between two boundaries within the same array?

In Experiment 1, it was found that when texture segregation is based on hue and form (circle, square) varies irrelevantly across the array, there is no interference. However, when segregation is based on form and hue varies irrelevantly, there is severe interference, even when the hue levels that vary are harder to discriminate than the form differences. One interpretation of these findings is that hue differences have a more important status than form differences when within-region similarity is calculated during texture segregation. The procedures employed in this experiment will permit an examination of whether hue differences also have a privileged status when between-region differences are processed.

In Experiment 3, observers saw arrays that contained one or two boundaries. For single-boundary arrays, texture was defined either by hue or by form differences (control arrays). For two-boundary arrays, one boundary was defined by hue differences and the other by form differences (ambiguous arrays). The discriminability of hue was varied in hue control and in ambiguous arrays in order to track the observers' preference for one boundary over the other across levels of hue discriminability. If there was dominance of one type of property difference over the other, then one would expect to see a high level of choice for a particular boundary type and that choice to be unrelated to the relative discriminabilities of the component hue and form differences. If there was no dominance of one type of property difference over the other, then one would expect to see the observers choose one type of boundary as long as the differences that defined it were more discriminable, and then switch to the other type once those differences became more discriminable.

\section{Method}

\section{Observers}

Eight Yale undergraduates were paid for their participation in this experiment. All had normal or corrected vision and took part in one experimental session lasting approximately $13 / 4$. 


\section{Procedure}

All aspects of the procedure were identical to those of Experiment 1 . In the instructions to observers, it was noted that some arrays may contain two boundaries and that they were to respond according to which one seemed to stand out more.

\section{Stimulus Construction and Presentation}

The stimuli were 35-mm slides of 36-element arrays that varied in hue and geometric form (circle, square), as in Experiment 1. In this experiment, eight hues from the Munsell color system were used: H1 (10R 4/12), H2 (7.5R 4/12), H3 (5R 4/12), H4 (2.5R 4/12), H5 (10RP 4/12), H6 (7.5RP 4/12), H7 (5RP 4/12), and H8 (2.5RP 4/12). Two forms were used: circle and square.

Slides were constructed and presented in a manner identical to that of Experiment 1. The viewing size of individual elements and of the entire array was also the same as that in Experiment 1.

\section{Stimulus Conditions}

There were two major types of stimulus arrays for this experiment; examples are depicted in Figure 3.

Single-boundary (control) arrays. The control arrays for this experiment were similar to those of Experiment 1 in that each array allowed for segregation according to either hue or form. Hue control arrays (Figure 3a) differed in the particular hue difference that produced texture. For this experiment, there were seven levels of hue discriminability varied in hue control arrays: 1 Munsell step (H1 vs. H2), 2 steps (H1 vs. $\mathrm{H} 3$ ), 3 steps (H1 vs. $\mathrm{H} 4), 4$ steps (H1 vs. H5), 5 steps (H1 vs. H6), 6 steps (H1 vs. H7), and 7 steps (H1 vs. H8). For each of these hue-discriminability levels, there was one stimulus array made for which the constant level of form was circle, and one for which it was square.

All form control arrays (Figure 3b) contained a difference of circle versus square elements. There were an equal number of form control arrays made using each of Hues $\mathrm{H} 2$ through $\mathrm{H} 8$ as the constant level of hue.

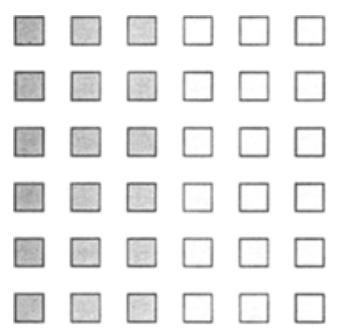

(a)

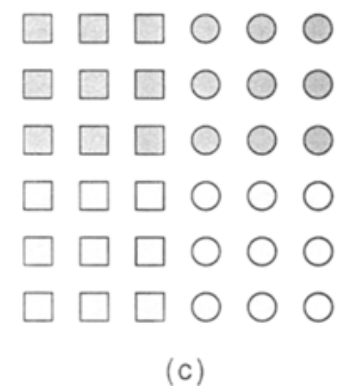

Figure 3. Examples of textured arrays containing single boundaries defined by (a) hue or (b) form (circle vs. square) differences, and (c) arrays containing two boundaries, one based on hue and the other on form.
Two-boundary (ambiguous) arrays. The second type of array (Figure 3c) was one for which the boundary judgment could be based on either one of two potential boundaries. In all ambiguous arrays, one of the potential boundaries was based on hue and the other on form differences. There were seven levels of ambiguous arrays, corresponding to the discriminability of elements that produced the hue boundary. These seven levels (1 step through 7 steps) were identical to the levels of discriminability for the hue control arrays. For all of the ambiguous arrays, the form boundary was produced by differences in circle versus square elements. For half of the ambiguous arrays, the hue boundary was horizontal and the form vertical, and for the remaining half the reverse was true.

\section{Experimental Design}

Forty-two different stimulus arrays (14 hue control, 14 form control, and 14 ambiguous) were used in this experiment. For hue control and ambiguous arrays there were two stimuli for each of the seven hue-discriminability levels. For form control arrays there were two stimuli for each of the hues, $\mathrm{H} 2$ through $\mathrm{H} 8$.

Each observer was presented with 21 blocks of trials, with the first block considered as practice. In each block, all 42 possible stimuli were presented once, and the order of presentation was randomized.

\section{Results and Discussion}

As in the previous two experiments, errors were low in this experiment (mean proportion of errors $=.05$ ) and were primarily found in the 1- and 2-step hue control arrays. ${ }^{3}$ For control arrays, the other experiments were followed in including only RTs for correct responses in further analyses. The raw RT data for each observer's correct responses were combined across blocks for each control-array subtype, and then median RTs were calculated. We did not analyze RTs for ambiguous arrays because of two factors: the RT data were highly variable and median RTs were not always available for all possible ambiguous-array subtypes for every observer. The latter factor ruled out the possibility of subjecting the data to the usual analyses. For ambiguous arrays, mean proportion of choice across blocks was calculated for a given type of boundary (i.e., hue or form) at each of the seven levels of hue discriminability for each observer.

\section{Control Analyses}

To establish the level of hue discriminability(ies) that equaled that of form discriminability for control arrays, correlated $t$ tests were performed comparing each observer's median RT for form control with his/her median RT for each of the seven hue control subtypes (i.e., 1 step through 7 steps). These data are depicted in Figure 4. The $t$ tests revealed that the mean RT for form control was equivalent to that for hue control at $3,4,5,6$, and 7 steps of hue discriminability. Furthermore, the mean RT for form control was significantly faster than that for Hue Control 1 step $(p<.002)$, as well as that for Hue Control 2 steps $(p<.002)$. The findings for the ambiguous arrays will be intepreted in light of these results.

\section{Ambiguous Analyses}

To determine whether the observers' responses for ambiguous arrays were dominated by choice for one dimen- 
sion over the other, their mean proportion of choices for each type of boundary at each of the hue-discriminability levels was subjected to a subjects $x$ type of boundary (hue, form) $\times$ hue-discriminability steps $(1,2,3,4,5,6,7)$ ANOVA. This analysis revealed a significant interaction (see Figure 5) of type of boundary and hue discriminability $[F(6,42)=16.65, p<.0001]$. Post hoc analyses of this interaction revealed that for Hue-Discriminability Steps 1 through 5 , there was a greater proportion of choice for form, relative to hue boundaries $(p<.01)$. For HueDiscriminability 6 steps, choice for hue and form boundaries was equal. Finally, for Hue-Discriminability 7 steps, there was a greater proportion of choice for hue, relative to form boundaries $(p<.05)$.

These data are not consistent with predictions made earlier suggesting that if dominance did occur, hue should be the more dominant dimension. In fact, if we adopt the stringent criterion of looking for equivalent choice performance as soon as form control is of equal discriminability to hue control (here, this occurred at 3 steps), then we see that the observers chose significantly more form than hue boundaries and continued to do so until the huediscriminability level of 6 steps. This may lead one to conclude, contrary to predictions, that form dominates in preattentive segregation. However, if we test, using correlated $t$ tests, whether choice for form arrays was significantly greater than chance level $(.50)$, we find that it was $(p<.02)$ for Hue-Discriminability Levels 1 through 3 , but not for Levels 4 through 7. Although choice for form was significantly greater than choice for hue at Discriminability Level 3 steps (where, according to control RT data, form and hue differences were equally discriminable), a downward trend was beginning at this point. Thus, a more parsimonious account of these data, consistent with Beck's (1982) model, is that the observers were choosing boundaries based on the discriminability of the component dimensions in a given array.

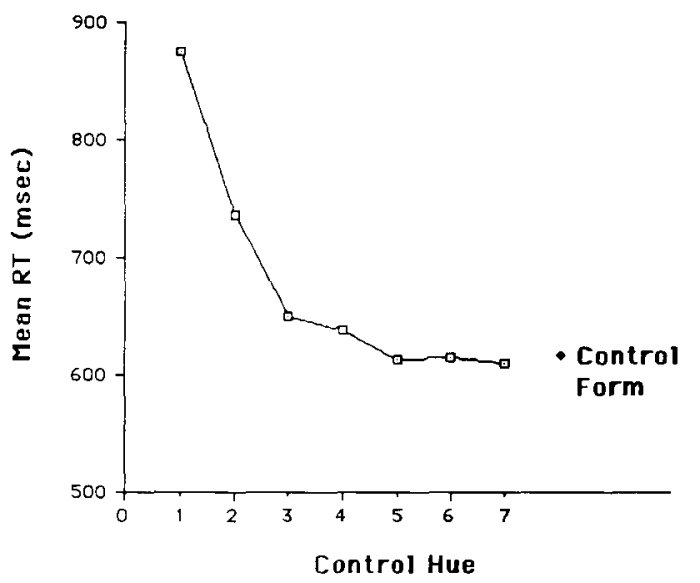

Figure 4. Mean reaction time (in msec) for hue control arrays at each of the seven levels of hue discriminability and for form (circle/square) control arrays.

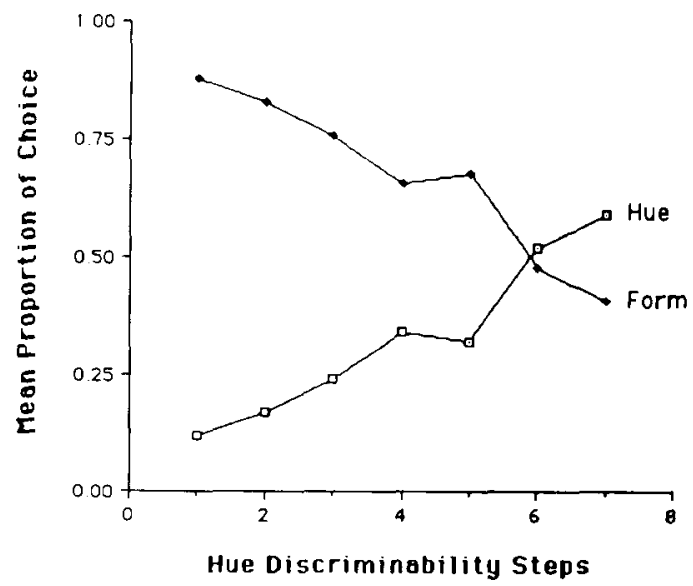

Figure 5. Mean proportion of choice for hue and form (circle, square) boundaries as a function of hue-discriminability level of the hue boundary in ambiguous arrays.

\section{EXPERIMENT 4}

This experiment was a replication of Experiment 3 using the forms (straight, curved) of Experiment 2. Given the findings of Experiment 3, in which the prototypical forms circle and square were used, evidence of dimensional dominance was not expected when the new forms were combined with the same hue differences.

\section{Method}

All aspects of the procedure, stimulus construction and presentation, and experimental design were identical to those of Experiment 3, with the exception that the novel forms (straight, curved) of Experiment 2 were used to produce form differences.

\section{Observers}

Ten St. Francis Xavier undergraduates, different from those run in the earlier experiments, were paid for their participation in this experiment. All had normal or corrected vision and participated in a session that lasted approximately $1 \frac{1}{6} \mathrm{~h}$.

\section{Results and Discussion}

Error rate was low in this experiment (mean proportion of errors $=.04$ ) and errors were confined to the most difficult hue control arrays ( 1 and 2 step). Thus, only RTs for correct responses were subjected to further analyses. The calculation of median RTs for each observer followed the pattern of Experiment 3.

\section{Control Analyses}

To assess when the discriminability of form (straight, curved) was equivalent to hue, correlated $t$ tests compared each observer's median RT for form control with his/her median RT for hue control at each of the huediscriminability steps (i.e., 1-7). Figure 6 presents these data in graphic form. These analyses indicated that form differences were equivalent to hue differences for hue control 2-step arrays. They were significantly faster than hue 
control 1-step differences $(p<.02)$ and were significantly slower than hue control 3- through 7-step differences. The findings for ambiguous arrays will be interpreted in light of these data.

\section{Ambiguous Analyses}

The observers' mean proportions of choice for hue or form boundaries at each level of hue discriminability were subjected to a subjects $\times$ type of boundary (hue, form) $x$ hue-discriminability steps (1-7) ANOVA. This analysis revealed a main effect of type of boundary that was qualified by an interaction between type of boundary and hue discriminability $[F(6,54)=53.98, p<.0001]$. This interaction is depicted in Figure 7. Post hoc analyses of this effect revealed that for Hue-Discriminability Steps 1 and 2, there was a greater proportion of choice for form, relative to hue arrays. In contrast, for Hue-Discriminability Steps 3 through 7 , there was a complete switch in boundary choice. Here, observers chose significantly more hue, relative to form boundaries. Figure 7 nicely illustrates that the switch from choice of form to hue boundary occurred between 2 and 3 hue-discriminability steps. This coincides exactly with the control data portrayed in Figure 6, which shows that the median RT for form control crosses the hue control curve just following 2 hue-discriminability steps. These findings replicate those of Experiment 3, and together they provide strong support for the contention that when hue and geometric form are pitted against each other in a preattentive boundary-judgment task, there is no dominance of one boundary type over the other. Rather, it appears that the observers' judgment of boundary was based on the boundary that was produced by the most discriminable difference.

\section{EXPERIMENT 5}

In this experiment, the issue of whether there would be dimensional dominance when hue was pitted against

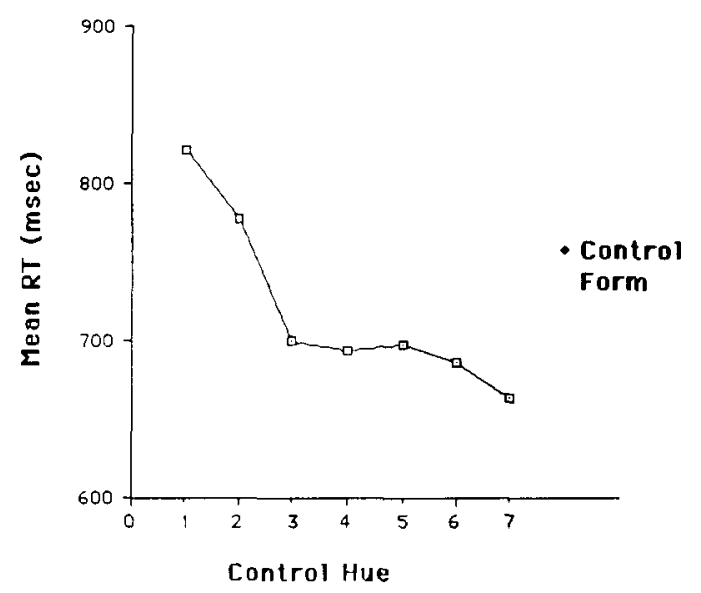

Figure 6. Mean reaction time (in msec) for hue control arrays at each of the seven levels of hue discriminability and for form (straight/curved) control arrays.

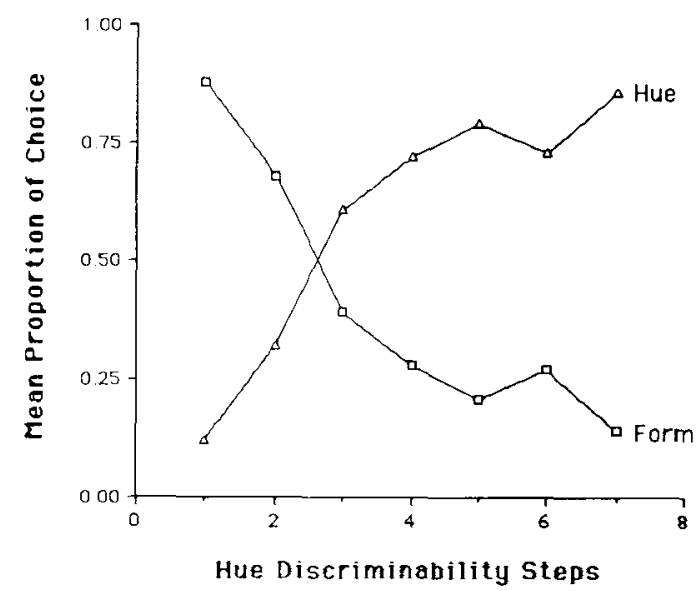

Figure 7. Mean proportion of choice for hue and form (straight/ curved) boundaries as a function of hue-discriminability level of the hue boundary in ambiguous arrays.

line orientation (horizontal, vertical) in ambiguous stimulus arrays was addressed. Callaghan et al. (1986) reported symmetric interference of texture segregation when within-region similarity was lowered by random variation of each of these component dimensions. Thus, there was no indication from existing preattentive research using these dimensions to suggest that one dimension may dominate over the other. Nonetheless, it was considered to be important to verify these predictions with an empirical test.

\section{Method}

The procedure, stimulus construction and presentation, and experimental design were similar to those of Experiment 3. The only exceptions were that the line orientations horizontal $(\mathrm{H})$ and vertical (V) replaced the forms circle and square when form differences defined the boundary, and the stimuli were constructed (see Figure 8) such that nine-element quadrants differed from the background in either hue or line orientation. For control arrays, single boundaries were defined by differences in hue (hue control, Figure 8a) or line-orientation (orientation control, Figure $8 \mathrm{~b}$ ) dimensions. For ambiguous arrays (Figure $8 \mathrm{c}$ ), two boundaries were possible, one defined by hue differences and the other by orientation differences. The observers were instructed to choose the different quadrant as quickly and accurately as possible. It was noted that, for some arrays, two quadrants may appear to be different, and that in this case they were to choose the one that stood out as most different.

\section{Observers}

Ten St. Francis Xavier undergraduates were paid for their participation in this experiment. None had participated in previous experiments of this kind. All had normal or corrected vision and participated in one session lasting approximately $1 * \mathbf{~ h}$.

\section{Results and Discussion}

The errors in this experiment were infrequent (mean proportion of errors $=.03$ ) and were found only in the most difficult hue control array (1 step). Thus, only RT data for correct responses were included in further anal- 
yses. Each observer's median RTs for each array subtype were calculated in the same manner as those in Experiment 3.

\section{Control Analyses}

To establish when orientation control discriminability equaled that of hue control, correlated $t$ tests were conducted on the observers' median RT for orientation control and each discriminability level of their hue control arrays (i.e., 1-7). The data relevant to these analyses are plotted in Figure 9. The analyses indicated that the discriminability of orientation control equaled that of hue control at Hue-Discriminability Level 2 steps. The mean RT for orientation control was significantly faster than Hue Control 1 step $(p<.01)$, and was slower than Hue Control 3 through 7 steps $(p<.01)$.

\section{Ambiguous Analyses}

The observers' mean proportion of choices for each boundary type were subjected to a subjects $x$ type of boundary (hue, orientation) $\times$ hue discriminability (1-7) ANOVA. These data are plotted in Figure 10. This analysis revealed significant main effects of type of boundary and hue discriminability. The main effects are qualified by a two-way interaction $[F(6,54)=143.41, p<.0001]$. Post hoc analyses of this interaction showed that observers chose significantly more $(p<.01)$ orientation boundaries at Hue-Discriminability Steps 1 and 2, and significantly more $(p<.01)$ hue boundaries at Hue-Discriminability Steps 3 through 7. Comparison of Figures 9 and 10 illustrates that the observers' switch from choice for orientation to hue boundaries coincided with the point at which

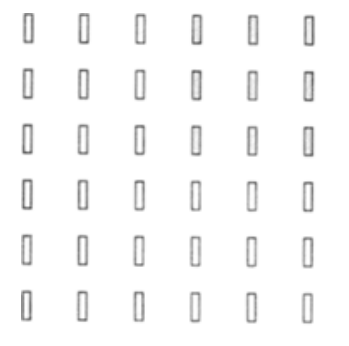

(a)

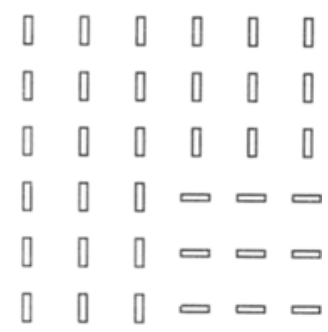

(b)

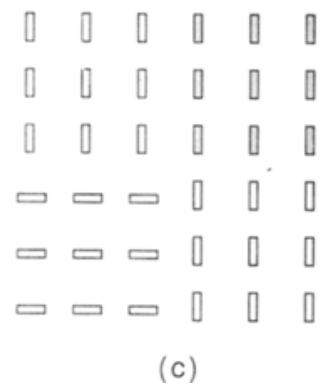

Figure 8. Examples of arrays containing single boundaries based on (a) hue or (b) line orientation, and (c) arrays containing two boundaries, one based on hue and the other on orientation.

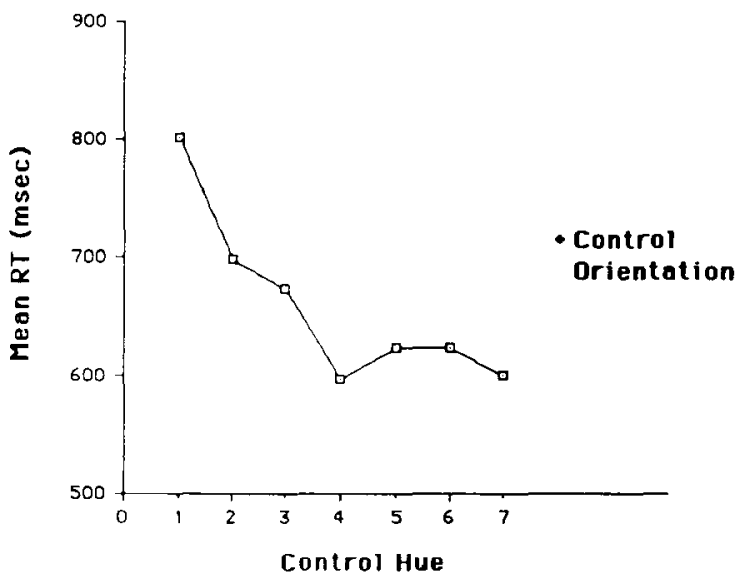

Figure 9. Mean reaction time (in msec) for hue control arrays at each of the seven levels of bue discriminability and for orientation control arrays.

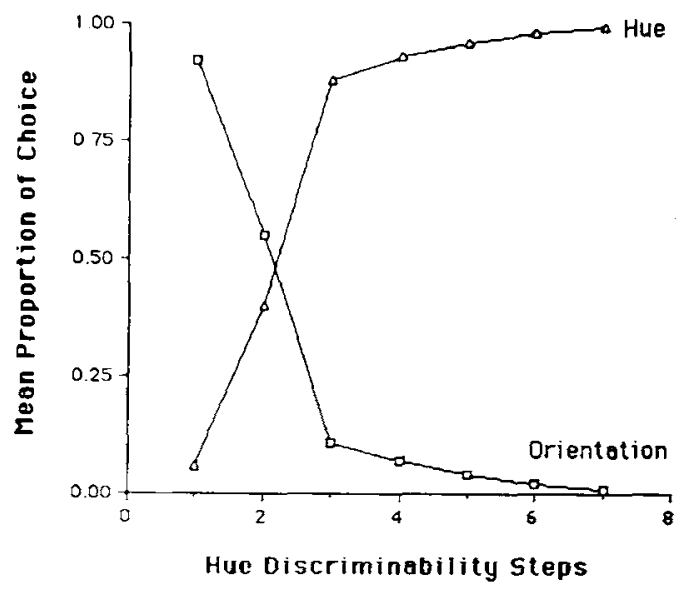

Figure 10. Mean proportion of choice for hue and orientation boundaries as a function of hue-discriminability level of the hue boundary in ambiguous arrays.

orientation control discriminability equaled that of hue control (i.e., 2 steps). Like the data of Experiment 4, these results strongly imply that there is no dimensional dominance in the preattentive process of texture segregation. Experiments 3 and 4 established this for the dimensions hue and geometric form, and this experiment confirms the lack of dominance for hue and line orientation.

\section{GENERAL DISCUSSION}

The purpose of these experiments was twofold: (1) to solidify the claim made by Beck and others (Beck, 1982; Callaghan et al., 1986; Enns, 1986; Olson \& Attneave, 1970 ) that within-region similarity is an important copponent of the texture-segregation process, and (2) to establish a procedure that could be used to assess whether certain dimensional differences, all previously confirmed to produce easy segregation in single-boundary arrays, 
would dominate over others when an observer was presented with a more complex array containing two potential boundaries.

\section{Within-Region Similarity}

In relation to the first aim, the results of Experiments 1 and 2 corroborate the findings of earlier studies by the author (Callaghan, 1984; Callaghan et al., 1986) for stimuli formed from the combination of hue and geometric form dimensions. In the present experiments, when withinregion similarity was reduced relative to control arrays by randomly varying the levels on an irrelevant dimension, strong interference of segregation occurred, but this interference was asymmetric. When boundaries were based on hue and form varied irrelevantly in low-similarity arrays, segregation responses were not hampered. This was true whether the forms that varied were circles versus squares or straight versus curved shapes, and also whether the forms were harder or easier to discriminate than the hues. In contrast, when boundaries were based on form differences and hue levels varied randomly across the array, segregation was impaired, regardless of whether the hue levels that varied were hard or easy to discriminate.

The finding that segregation is impaired when a second, irrelevant dimension varies challenges the strong assumption made by Treisman (Treisman \& Gelade, 1980; Treisman \& Paterson, 1984; Treisman \& Souther, 1985) that feature information is completely independent at this early stage of processing. The implications of the revised feature-integration model for texture segregation have not been specifically outlined by Treisman and Gormican (1988). Given Treisman's visual-search pop-out findings and the physiological data that she refers to as support for her results, it is highly probable that there is a first stage of independent feature registration by the visual system. However, given the evidence that shows interference of texture segregation when irrelevant feature information is varied (Beck, 1982; Callaghan, 1984; Callaghan et al., 1986), we must conclude that the process of textural segregation involves some narrowing of the attentional focus to allow for interdimensional interaction. Texture segregation is more than simple, independent registration of features.

Beck (1982) has proposed a model that can effectively accommodate the interference effects. This model is specifically directed to texture-segregation findings. Beck suggested that there is first a detection of features across the visual display, followed by a detection of the total differences (i.e., a summation of differences across all preattentively discriminable dimensions) between textural elements in neighboring spatial regions. Finally, a decision about where the regions of texture lie (or where the boundary lies) is made on the basis of the magnitude and spatial distribution of difference signals. If, as in our lowsimilarity arrays, strong between-region difference signals are subdued by local within-region differences, then the decision of where the boundary lies will be slowed, perhaps until such time as a difference threshold is reached. The only problem with Beck's model, given our results, is that there is no apparent mechanism that can explain why some differences (hue) can interfere to the exclusion of others (geometric form), especially when those others are of equal discriminability. A check of individual observers' performance revealed that $25 \%$ of the observers in Experiment 1 and 50\% of the observers in Experiment 2 did show symmetric interference; that is, hue interfered with form and form interfered with hue. Thus, the data for some of the subjects in the present study fits well with predictions from Beck's model. Nonetheless, the fact that the same pattern of overall asymmetry was shown in the present texture-segregation task as has previously been reported in visual search by Pashler (1988) and in the attentional literature (Garner, 1983; Gottwald \& Garner, 1972; Schroeder, 1976) suggests that the effect is a real one in need of further examination.

Treisman and Gormican (1988, p. 40) offered an interesting speculation that may relate to the present asymmetric results. They suggested that feature analysis in visual search seems to take place automatically in a number of different dimensions, and they stated that the particular feature codes that are activated for each search situation has not yet been established. Given the present findings, it may be that the code for hue is automatically activated (hence, potentially interfering) when observers judge form boundaries, but the code for form is not activated (hence, not able to interfere) when observers judge hue boundaries. Empirical evidence from a convergence of tasks will be necessary to assess these hypotheses.

\section{Dominance of Dimensions}

The second aim of the present experiments was to come a bit closer to real-world experience for the viewer and to see whether boundaries based on differences in one dimension would dominate over those based on another dimension. Prior to these experiments, there was the finding that a small number of physical properties (e.g., brightness, hue, line orientation, line length, curvature, form, closure, presence of line terminators) appeared to successfully produce texture segregation. From this information alone, it could be concluded that, given equal discriminability, there should be no dominance of one simple property difference over another. However, there was also evidence that segregation could be interfered with by varying certain of these simple properties. In most cases, the interference was symmetric (hue and brightness, hue and horizontal/vertical lines, hue and right/left diagonals), but in some cases it was asymmetric (hue and geometric form, hue and horizontal/left diagonal lines). One implication of the finding of asymmetric interference is that there is indeed property dominance in texture segregation, and it is to a test of this possibility that Experiments 3-5 were focused. The results of the present experiments unequivocally show that when the property of hue was pitted against that of form or line orientation there was no dominance. Rather, the results are perfectly in tune with Beck's (1982) model suggesting that the major 
mechanism involved in texture-segregation decisions is one based on the discriminability of differences between and within the texture regions.

\section{Preattention: Distinct Stage or Continuum of Processing?}

This leads us to the question, first posed by Beck (1982) and recently reassessed by Treisman and Gormican (1988), concerning whether the concept of preattention as a qualitatively distinct processing stage is still a theoretically useful one. The interference findings reported here and elsewhere (e.g., Beck, 1982) pose problems for the proposal that feature information is independent in the preattentive stage; there could be no interference unless there was some form of interaction of feature information. Admittedly, the results of the present experiments showing no dominance of property differences can be handled by Treisman's early feature-integration model, which calls for a preattentive/attentive distinction (Treisman \& Gelade, 1980). However, the pattern that emerges over the five experiments reported here favors a continuum view of attention (Beck, 1982; Callaghan et al., 1986; Treisman \& Gormican, 1988).

Why do interference effects emerge in the present experiments, and not in earlier ones? In both the interference and the dominance paradigms, the visual displays (i.e., low-similarity and ambiguous arrays) were more complex, hence more like the real visual world, than those used in traditional texture-segregation (e.g., Beck, 1966; Treisman \& Gelade, 1980) and visual-search (e.g., Treisman \& Gelade, 1980) paradigms. In the traditional paradigms, the typical textured display has only a single element or a region of elements (i.e., the present control arrays) embedded in a homogeneous background. It may be that as we complicate the visual display, we get a different picture of the nature of the process.

Treisman and Paterson (1984) advise us, following Garner, Hake, and Eriksen (1956), to look for a general pattern that emerges from experiments that employ a variety of procedures and stimuli. It appears that as the stimuli become more complex in studies of texture segregation, the general pattern suggests that the old view of preattention is no longer useful. Judging from the fact that our "complex" arrays are still very simple when compared with the view from our windows, there is still much to be explored in the realm of texture segregation.

\section{REFERENCES}

BEck, J. (1966). Effect of orientation and of shape similarity on perceptual grouping. Perception \& Psychophysics, 1, 300-302.

Beck, J. (1967). Perceptual grouping produced by line figures. Perception \& Psychophysics, 2, 491-495.
Beck, J. (1972). Perceptual grouping and peripheral discriminability under uncertainty. American Journal of Psychology, 85, 1-19.

BECK, J. (1974). Relation between similarity grouping and peripheral discriminability. Joumal of Experimental Psychology, 102, 1145-1147.

Beck, J. (1982). Textural segmentation. In J. Beck (Ed.), Organization and representation in perception (pp. 285-317). Hillsdale, $\mathrm{NJ}$ : Erlbaum.

Callaghan, T. C. (1984). Dimensional interaction of hue and brightness in preattentive field segregation. Perception \& Psychophysics, 36, 25-34.

Callaghan, T. C., Lasaga, M. I., Garner, W. R. (1986). Visual texture segregation based on orientation and hue. Perception \& Psychophysics, 39, 32-38.

EnNs, J. (1986). Seeing textons in context. Perception \& Psychophysics, 39, 143-147.

GARNER, W. R. (1983). Asymmetric interactions of stimulus dimensions in perceptual information processing. In T. J. Tighe \& B. E. Shepp (Eds.), Perception, cognition, and development: Interactional analyses (pp. 1-38). Hillsdale, NJ: Erlbaum.

GARNer, W. R., HAKe, H. W., ERIKSEN, C. W. (1956). Operationism and the concept of perception. Psychological Review, 63, 149-159.

GotTwald, R. L., Garner, W. R. (1972). Effects of focusing strategy on speeded classification with grouping, filtering, and condensation tasks. Perception \& Psychophysics, 11, 179-182.

JuLESz, B. (1975). Experiments in the visual perception of texture. Scientific American, 232, 34-43.

JuLESZ, B. (1981). Textons, the elements of texture perception, and their interactions. Nature, 290, 91-97.

Khaneman, D. (1973). Attention and effort. Englewood Cliffs, NJ: Prentice-Hall.

Neisser, U. (1967). Cognitive psychology. New York: AppletonCentury-Crofts.

Olson, R. K., ATtNeAve, F. (1970). What variables produce similarity grouping? American Journal of Psychology, 83, 1-21.

PAshier, H. (1988). Cross-dimensional interaction and texture segregation. Perception \& Psychophysics, 43, 307-318.

SCHROEDER, R. (1976). Information processing of color and form. Unpublished honor's thesis, University of Oregon, Eugene.

Treisman, A. M. (1982). Perceptual grouping and attention in visual search for features and for objects. Joumal of Experimental Psychology: Human Perception \& Performance, 8, 194-214.

Treisman, A. M., Gelade, G. (1980). A feature-integration theory of attention. Cognitive Psychology, 12, 97-136.

Treisman, A. M., Gormican, S. (1988). Feature analysis in early vision: Evidence from search asymmetries. Psychological Review, 95, $15-48$.

Treisman, A. M., \& Paterson, R. (1984). Emergent features, attention and object perception. Joumal of Experimental Psychology: Human Perception \& Performance, 10, 12-31.

Treisman, A. M., Souther, J. (1985). Search asymmetry: A diagnostic for preattentive processing of separable features. Journal of Experimental Psychology: General, 114, 285-310.

\section{NOTES}

1. This type of array has been called orthogonal in previous experiments by this author (Callaghan, 1984; Callaghan et al., 1986).

2. Equal area was ensured by cutting templates for these forms from uniform bond paper and weighing them on a Mettler balance.

3. Errors were possible only for control arrays.

(Manuscript received September 6, 1988; revision accepted for publication March 23, 1989.) 